\title{
Análise auditiva do \\ Quartetto per archi in \\ due tempi (1955) de \\ Bruno Maderna
}

Anna Rita Addessi

(Universidade de Bolonha/ltália)'

Tradução: Rosane Cardoso de Araújo (UFPR)

${ }^{1}$ Autorização da tradução: Revista "Musicae Scientiae". 
RESUMO: O presente estudo sobre quarteto de Maderna visa comparar a "macroforma" (a grande estrutura de uma peça musical) percebida por indivíduos por meio da audição da obra em tempo real, e as "macroformas" percebidas por analistas/especialistas da música. O estudo também analisa a correlação entre "macroforma" e percepção de tensão/relaxamento. Dois grupos de indivíduos tomaram parte no experimento: 25 não-músicos e 32 músicos. O experimento foi realizado em Bolonha e em Edimburgo. Foi utilizado um programa de computador especial, que permitiu que cada sujeito ao ouvir a obra através de fones de ouvido, indicasse os pontos de divisão percebidos de tensão/relaxamento, enquanto escuta em tempo real. O experimento durou cerca de 60 minutos e envolveu várias etapas e tarefas. Depois de uma escuta inicial, que serviu para a familiarização com a peça e com o programa de computador, os sujeitos tiveram de ouvir a peça novamente e identificar suas seções principais. Eles, então, tiveram de indicar os critérios que usados na formulação de suas soluções. Na escuta seguinte, os participantes indicaram as maiores zonas de tensão e relaxamento percebidas. As macroformas percebidas pelos indivíduos foram comparadas com as 4 macroformas indicadas pelos especialistas em análise musical com base em processos auditivos e não em teorias analíticas. Os dados revelaram maior correlação entre as macroformas percebidas pelos sujeitos em apenas 2 macroformas indicadas pelos analistas, e também a correlação entre as macroformas e tensão/relaxamento percebidas pelos participantes. As diferenças entre os músicos e não músicos e dos grupos italianos e de Edimburgo não foram significativas.

PALAVRAS-CHAVE: macroforma; percepção, quarteto de Maderna

ABSTRACT: The present study on Maderna's quartet aims to compare the "macroform" (the large-scale structure of a musical piece) perceived by subjects while listening to the piece in real time, and the "macroform" as perceived by analysts. The study also examines the correlation between "macroform" and perception of tension/relaxation. Two groups of subjects took part in the experiment: 25 non-musicians and 32 musicians. The experiment was carried out both in Bologna and in Edinburgh. We made use of a special computer program, which allows each subject to listen to a piece through head-phones and indicate the points of division and the tension/relaxation perceived while listening in real time. The experiment lasted approximately 60 minutes and involved various steps and tasks. After an initial listening which served to become familiar with the piece and with the computer program, the subjects had to listen to the piece again and identify its main sections. They then had to indicate the criteria they used in formulating their solutions. In the next listening they had to indicate the largest zones of tension and relaxation they perceived. The macroforms perceived by the subjects were compared with 4 macroforms indicated by professional analysts based on listening rather than on analytical theories. The data showed higher correlation between macroforms perceived by the subjects and only 2 macroforms indicated by the analysts, and correlation between the macroforms and tension/relaxation perceived by subjects. The differences between musicians and non musicians and Italian and Edinburgh groups were not significant.

KEYWORDS: macroform, perception, Maderna's quartet 
People very often ask me questions about form. They expect me to talk about mathematical formulae, a bit like I do with spectrum; it's easy with spectrum, but not with time. I find it impossible to use the same form for different pieces: every musical work is a new experience of time. It's for this reason that I can't explain it beforehand...because for me, explaining the form of a piece is the same as listening to it. Talking about form when we are dealing with listening, is simply a pleonasm....it doesn't add anything else. (Tristan Murail 2002)

Neste artigo é apresentado um estudo exploratório sobre o quarteto de Maderna com o de objetivo investigar as relações entre as macroformas percebidas pelos indivíduos na escuta desta obra pós-tonal e as macroformas da mesma peça indicadas por analistas profissionais, com base na escuta e não em teorias analíticas. Esta investigação é parte de um projeto realizado na Universidade de Bolonha, que trata da análise e percepção de "macroforma" na música pós-tonal, ou seja, o problema da forma global de uma peça. O projeto tem como foco principalmente quartetos de cordas do século 20 e tem sido conduzido por meio da utilização de análises de partituras e análises auditivas (Addessi \& Caterina 2000, 2005, Baroni 2003) ${ }^{2}$.

O foco deste artigo é investigar se a percepção de “pistas" (Deliège 2001) e tensão/relaxamento (Imberty 2005) poderiam afetar a análise auditiva da macroforma de uma peça de música pós-tonal, e se esta análise poderia ser utilizada como um critério "explícito" para a análise de música pós-tonal (Cross, 1998). Antes de mais nada, uma visão geral de macroforma, segmentação e tensão/relaxamento é introduzida, a fim de identificar os conceitos básicos subjacentes ao presente estudo. Na sequencia será examinado brevemente o quarteto de Maderna e os métodos e os resultados serão apresentados e discutidos.

\footnotetext{
${ }^{2}$ The project was developed inside the post-tonal research team of GATM-Gruppo di Analisi e Teoria Musicale, coordinated by Mario Baroni, from 1998 to 2003. The Author collected the data illustrated in this paper in 2001 and was funded by the University of Bologna.
} 


\section{Macroforma e segmentação}

O termo "macroforma" e o conceito identificado para este termo necessitam ser cuidadosamente definidos, uma vez que eles não são sempre usados da mesma maneira. O termo "macroforma" se refere a forma global de uma peça, isto é, a divisão da peça em suas partes maiores, com referência à sua estrutura geral. Na literatura musicológica diversos termos utilizados parecem ter um sentido similar, mas na verdade têm significados que são às vezes muito diferentes: por exemplo, "large dimensions" (La Rue, 1970), de “large scale form” (Levinson, 1997), ou “large-scale structure" (Lerdhal \& Jackendof 1983, Lalitte et al. 2009). Imberty (1993) usa o termo "macroestrutura", que tem origem no campo da linguística, mas que é aqui considerado do ponto de vista da percepção: um esquema "para a estruturação de tempo, onde são organizados eventos de som, a priori, de acordo com regras elaboradas a partir de mecanismos perceptuais envolvidos na detecção de mudanças dentro do continuum do som" (1981, p. 90) ${ }^{3}$.

Neste estudo, o uso dessa palavra (macroforma) decorre, basicamente desse conceito de macroestrutura, mas foi necessário distinguir entre o conceito de “divisão em partes”, que só identifica a macroforma, a percepção de "mudança” e de "descontinuidades locais" que são definidas com o termo "segmentação". Embora o termo segmentação seja usado como um sinônimo para a divisão em partes, a segmentação não é um critério suficiente para identificar as partes principais de uma peça (ou seja, sua macroforma) pois a percepção de uma "forte descontinuidade local" não produz necessariamente uma divisão de termos da peça como um todo. Uma pausa muito longa, por exemplo, pode dar origem à percepção de uma segmentação, mas isso não constitui necessariamente uma seção independente separada. As razões por trás das escolhas utilizadas para a segmentação variam consideravelmente, mas em geral incluem: repetição (Ruwet, 1966), alteração ou descontinuidade (Imberty, 1981), agrupamentos rítmicos (na

\footnotetext{
3 "scheme for structuring time, where sound events are arranged a priori according to rules drawn from the perceptual mechanisms involved in the detection of changes within the continuum of sound" (Imberty, 1981, p. 90).
} 
música metricamente organizada como apontam Lerdahl e Jackendoff, 1983) e os princípios de diferença e semelhança (Deliège 2001a, 2001b, 2007).

A análise de macroforma coloca problemas diferentes para a segmentação, especialmente para a memória. A questão é: quando chegar ao final da composição, podemos elaborar mentalmente uma possível divisão da peça em partes? E somos capazes de dizer o número exato de partes e identificá-las? Podemos identificar as partes, como resultado das suas qualidades e homogeneidade, ou com base em "descontinuidades locais"? Para os fins deste artigo, por conseguinte, o termo segmentação será usado apenas para indicar o ponto exato em que duas seções são separadas, um fenômeno local provocado pela presença de uma descontinuidade ou de um contraste que envolve um ou mais parâmetros do material musical (duração, dinâmica, densidade, timbre, registro, etc.). O termo macroforma, por outro lado, é utilizado para indicar o resultado de um processo de memorização que envolve a divisão de uma obra em seus principais componentes, nos quais cada parte possui a coerência estrutural e homogeneidade. Neste sentido, o conceito de macroforma utilizado neste estudo não coincide necessariamente com quaisquer hierarquias tonais ou rítmicas (como, por exemplo, no modelo Lerdhal e Jackendoff), ou com segmentações baseadas na percepção de ideias musicais (ver, por exemplo, a conceito de estruturas em grande escala em Lalitte et al. 2009). Do ponto de vista perceptível, a macroforma está mais próxima do conceito de “imprinting” proposto por Deliège (2001).

Dado que o objetivo principal deste estudo foi investigar a memorização de macroforma, que é a divisão de uma peça em seus maiores segmentos, os participantes do experimento não foram convidados a indicar os pontos de segmentação, mas sim os pontos de divisão das partes. Os sujeitos foram convidados a ouvir a mesma peça várias vezes, dividindo-a em partes principais por meio da indicação dos pontos de divisão entre uma parte e outra, oralmente. Para a música tonal, Deliège (1998) propôs um procedimento experimental particular, que é o uso da uma "linha mental" que testa a capacidade de memorizar a qualidade e a ordem de sucessão das partes identificadas em um trecho de música enquanto o sujeito escuta. Em nosso experimento, os participantes também foram convidados a respeitar a proporção entre as partes: 
seções mais longas de "linhas mentais" tiveram que corresponder a partes mais longas. Este procedimento impôs um limite de 4-8 partes em média. Através deste procedimento, portanto, os participantes da pesquisa foram solicitados a memorizar implicitamente as peças. A tarefa de divisão da obra nas partes principais, assegurou que o processo de segmentação não a atenção do ouvinte fosse guiada por descontinuidades e micro segmentações locais. A fim de tirar algumas conclusões sobre os critérios que levaram o ouvinte a dividir as partes, apresentamos algumas perguntas que visavam investigar como os ouvintes percebiam a "qualidade" das partes: eles foram convidados a descrever a qualidade de cada parte por eles determinada, tanto por meio de adjetivos quanto por meio de parâmetros de sons.

\section{A percepção de macroforma na música pós-tonal}

Uma última observação também deve ser feita sobre a diferença entre os critérios utilizados para a divisão em partes de uma obra pós-tonal e uma obra tonal. Estudos realizados por Deliège $(2001,2007)$ mostram como o ouvinte, quando confrontado com a música que não é organizada por estruturas tonais, tende a identificar certas qualidades de igualdade e de diferença que podem ser facilmente percebidas e memorizadas, permitindo ao ouvinte "pistas" (ou características proeminentes) que distinguem as partes da obra. Estas "pistas", que na música tonal são fornecidos pelas estruturas hierárquicas harmônicas, melódicas e rítmicas, também podem aparecer em música atonal, principalmente, como elementos superficiais ligados à dinâmica, velocidade, duração e timbre. Lerdhal (1989) sugere que na música atonal os critérios que constituem a hierarquia sejam baseados em parâmetros de timbre. A divisão em partes de uma peça tonal depende muito, no entanto, de regras formais (e culturais) - exposição, desenvolvimento, conclusão, coda, recapitulação, ponte, simetria, repetição, etc. Traços destas regras culturais podem, de fato, persistirem na música pós-tonal (Baroni 2003, Addessi e Caterina 2005, Imberty 2005, Lalitte et al. 2009). 


\section{Macroforma analisada e macroforma percebida}

As ligações entre a estrutura formal e processos psicológicos na música já foi amplamente discutido no âmbito das ciências cognitivas. Embora pertencendo a dois domínios diferentes (psicologia da percepção e teoria musical), as duas análises (análise da partitura e análise auditiva), possuem vários elementos em comum: a segmentação em trechos pequenos, médios ou grandes; a formação de agrupamentos; as organizações de hierarquias, e assim por diante. Cross (1998) colocou o problema do aspecto da relação entre a análise perceptivo-auditiva e análise musical como a diferença entre a psicologia da percepção - que estuda os fenômenos de percepção do ponto de vista cognitivo - e da percepção do ponto de vista de analistas musicais - orientada fundamentalmente para a confirmação de teorias musicais (definida por Cross como "folk psychology"). A função da psicologia da percepção poderia ser a de tornar explícito uma suposição perceptual em um trecho de análise musical, de forma discutível e verificável.

Nesta pesquisa, o objetivo, portanto, foi investigar a análise auditiva da macroforma a fim de distinguir as "regras" que poderiam ser usadas para a análise da partitura. Nos estudos experimentais preliminares foi investigada a percepção de várias macroformas em quartetos do século 20. Ao trabalhar nesse repertório homogêneo, o número de variáveis torna-se limitado e, portanto, fica mais fácil formular hipóteses plausíveis e gerais sobre os problemas de ouvir música póstonal.

Em Addessi e Caterina (2005) foi feita uma comparação entre as análises macroformal do quinto movimento do Quarteto de Cordas Op. 1 de G. Kurtág, por meio da colaboração de vários especialistas em análise musical, profissionais de diferentes universidades italianas, que verificaram as percepções de dois grupos de sujeitos (músicos e não músicos) ao ouvir a peça em tempo real. As análises de macroforma foram em grande parte realizadas com base na teoria Deliège de "sugestão de abstração" (cue abstraction), e na teoria de “domínios" propostos por Christopher Hasty (1981). Os resultados mostraram que as diferenças entre os procedimentos analíticos e de escuta não foram particularmente significativas no caso da análise da partitura que foi baseada em critérios explícitos extraídos de 
teorias relativas a determinados aspectos perceptuais de som (nomeadamente o modelo de abstração de "pistas").

A experiência que vamos apresentar a seguir baseia-se no segundo movimento do Quartetto per Archi in Due Tempi de Maderna, cujo objetivo foi o de investigar as relações entre as macroformas indicadas pelos analistas e as macroformas percebidas pelos sujeitos ao escutar a peça em tempo real. Neste experimento, os analistas foram convidados a usar modelos explicitamente perceptivos, de forma ampla para a memorização, a fim de arquivar suas análises de partitura ${ }^{4}$.

\section{Correlação entre macroforma e tensão/relaxamento}

De acordo com as hipóteses de vários estudiosos, a forma em grande escala de uma peça é assimilada pelo ouvinte através de sistemas de tensão e relaxamento (ver, por exemplo Imberty 1993, Bigand, Parncut, Lerdhal 1996). O conceito de tensão na música tem sido difícil de definir devido ao problema de descrever a experiência em si e de indicar as estruturas musicais geradoras de tensão. Na música tonal estes esquemas são ativados principalmente pela sintaxe tonal (ver, por exemplo, Meyer, 1956; Nielsen, 1989; Lerdahl 1987, 1996; Bigand 1993; Krumhansl 1996, 1997). De acordo com Imberty (2005) uma dupla função existe na memória musical que cria duas possibilidades de processar os dados percebidos mentalmente. Por um lado, há uma "memória informativa" que organiza a informação de som em sistemas sintáticos sejam eles tonais ou atonais, por outro lado, há uma "dinâmica de memória" ligada ao dividir o material sonoro ao longo do tempo. É neste segundo tipo de memória que os conceitos tais como tensão e relaxamento estão situados. Na música atonal, tal como no quarteto de Maderna, os regimes de tensão e relaxamento podem desempenhar um papel determinante, porque permitem que o ouvinte assimile a forma total da peça musical, mesmo se houver uma falta de pontos de referência tonais. Em

${ }^{4}$ Os primeiros resultados destes experimentos foram apresentados em Addessi \& Caterina (2002). 
pesquisa recente Lalitte et al. (2009) mostraram que a tensão e relaxamento afetam a percepção da estrutura em larga escala de uma peça de música atonal, durante a audição em tempo real.

Neste estudo, a estrutura temática e retórica do estilo tonal da peça original, que não é modificada, parece continuar a ter um papel determinante na percepção da forma e também na percepção da tensão/relaxamento. Em experiência anterior (Addessi \& Caterina, 2000) foi investigada a relação entre a memorização da macroforma e a percepção de tensão/relaxamento em relação à presença de estruturas tonais/atonais. Foram usadas três peças de música com uma distância gradual do sistema tonal, de Milhaud, para Maderna e finalmente para Webern. Os dados sobre tensão e relaxamento foram apenas parcialmente sustentados dentro do previsto, apontando que na parte mais tonal (Milhaud) o nexo de causalidade entre o ponto de divisões (de tensão e relaxamento) seria mais forte do que na peça onde a tonalidade é menos presente (Maderna) ou totalmente ausente (Webern). Na realidade, foi observado também que na obra de Webern a macroforma parece ter sido influenciada pela percepção de tensão e relaxamento, mais do que em Maderna. O estudo exploratório apresentado neste artigo faz uso do mesmo paradigma experimental, a fim de investigar se mais de uma vez a memorização da macroforma de uma obra pós-tonal pode ser influenciada pela alternância entre tensão e relaxamento experimentada pelo sujeito durante a audição da obra.

Foi buscada uma estratégia para formular uma definição da tensão, decorrente da necessidade de explicar aos ouvintes o que eles tinham que indicar. A solução foi pedir-lhes para indicar as zonas de tensão e relaxamento, movendo para cima do mouse (aumento de tensão) ou para baixo (relaxamento): as zonas de tensão foram caracterizadas pela instabilidade, como "inspirar" e as zonas de relaxamento foram sendo caracterizado por estabilidade, como "expirar". Foi pedido aos participantes para identificar somente as grandes zonas de tensão/relaxamento, e não as alterações menores de tensão/relaxamento. 


\section{Percepção musical de músicos e não músicos}

Neste estudo, procurou-se examinar se haviam diferenças no processo auditivo/perceptivo entre músicos e não-músicos. Esta variável tem sido cada vez mais levada em consideração em estudos experimentais sobre a percepção da música e envolve fatores sociológicos e antropológicos (aculturação musical); fatores psicológicos; fatores pedagógicos (formação musical); e fatores relacionados à prática instrumental e neurociências. Quanto à natureza da representação musical “muitas investigações perceptivas e cognitivas nessa área podem ser vistas como demonstrando que há, de fato, grandes áreas de concordância entre indivíduos sobre tais assuntos. Estas concordâncias, muitas vezes transcendem diferentes níveis de treinamento, e, às vezes, transcendem culturas" (Sloboda 2005, 165) ${ }^{5}$.

Em uma série de fases experimentais, Deliège $(2001,2007)$ mostra que os princípios perceptivos que estão na base do modelo de representação mental da música, durante a audição e em tempo real, ou seja, os princípios da formação de segmentação, categorização, esquematização e formação impressa, são comuns a ambos: músicos e não músicos. Bigand (2003), por exemplo, mostra como a sensibilidade a hierarquia tonal e harmônica de semelhança de acordes, bem como a capacidade de detectar a modulação, foram encontradas de forma semelhante em grupos com diferentes níveis de expertise musical. Estes resultados fornecem evidências de que os não-músicos conseguem interiorizar complexos sistemas musicais através da exposição passiva à música. Mesmo quando os nãomúsicos levaram mais tempo para resolver quebra-cabeças harmônicos, seus desempenhos nos processos de audição musical permaneceram altamente comparáveis às dos músicos.

Drake, Penel, e Bigand (2000), ao contrário, mostraram a grande capacidade de músicos (versus não-músicos) em sincronizar toques com uma sequência musical, extrair a hierarquia métrica de uma sequência, e assim, ter

\footnotetext{
5 "much perceptual and cognitive research in this area can be seen as showing that there are, indeed, large areas of agreement between individuals about such matters. These agreement often transcend different level of training, and sometimes, transcend cultures" (Sloboda 2005, 165).
} 
uma representação hierárquica mais complexa; os músicos têm uma representação mental mais elaborada e mais distante da superfície musical, mais organizada hierarquicamente e mais flexível. Os estudos de neurociências da música demonstraram que certas regiões do cérebro (corpo caloso, córtex motor, cerebelo) apresentam formas de adaptação a desafios extraordinários das exigências da performance musical (Pantev et al. 2001, Pascual-Leone 2001. Schlaug 2001, Habib Besson \& 2009).

Outros estudos tendem a salientar não tanto a capacidade maior ou menor dos músicos em tarefas musicais, mas as diferenças de percepção, devido à formação musical recebida. Sloboda (2005), por exemplo, conclui que, em tarefas de leitura musical a familiaridade com os caracteres visuais da notação musical poderia ser uma explicação suficiente da superioridade músicos. Bamberger (1995), mostra como a representação gráfica do agrupamento rítmico pode ser de um tipo de métrica (que se baseia no presente acento métrico da música ocidental) no caso de músicos, ou de um tipo de figura (que se baseia na proximidade/distanciamento dos acentos) no caso de não-músicos. Ela mostra como ambos os temas seguem uma lógica perceptiva, diferindo apenas em que, para um músico, esta lógica é determinada por modelos musicais métricos ocidentais aprendidos durante o treinamento.

Como se pode ver, os resultados obtidos variam de acordo com a capacidade do indivíduo e os processos cognitivos culturais. Tendo em vista que o foco da presente pesquisa é sobre a percepção de macroformas e tensão/relaxamento, observa-se que Imberty (1981) e Bigand (1997) sugerem que os processos psicológicos envolvidos na rede de tensão/relação musical experimentada pelos ouvintes em música tonal, não são fortemente influenciadas pela formação musical. Em nossos estudos anteriores sobre a percepção de macroforma na música pós-tonal, a diferença entre músicos e não-músicos foi demonstrada como insignificante. Não obstante, as diferenças na precisão da tarefa e na definição de motivações foram anotadas (Addessi e Caterina 2000). Dois grupos de indivíduos, músicos e não-músicos, também foram utilizados no experimento apresentado neste artigo. 


\section{O Quartetto per archi in due tempi de Maderna}

Quarteto foi escrito em 1955, encomendado pela prefeitura da cidade de Darmstad e dedicado ao amigo de Maderna, Luciano Berio. A estreia da obra foi realizada pelo quarteto Drolc, em 01 de junho de 1955, para a décima edição do Ferienkurse $^{6}$. Os críticos concordaram que, com este trabalho, Maderna havia chegado mais próximo às ideias pós-Webern e à prática composicional predominante nos círculos de pesquisa musical avançada dos anos 50. "A escrita complexa da partitura, juntamente com uma infinidade de marcas realizadas, presentes em praticamente cada nota, é prova do trabalho sério e do cuidado na composição do Quarteto, realizado na convicção de ter adquirido uma linguagem distinta e pessoal" (Russo, 2010, s/p) ${ }^{7}$. O processo de composição foi um trabalho extremamente difícil e Maderna comentou mais de uma vez sobre isso: "Foi um trabalho enorme, muito pior do que escrever as partes para uma obra orquestral", e novamente "eu acredito que com este trabalho eu tenha verdadeiramente encontrado meu caminho", e mais uma vez "tudo o que eu compus antes é velho kitsch"8 (Maderna apud Russo, 2010). Para Maderna, o trabalho era "saudável e positivo", "revolução na continuidade".

O segundo movimento, no qual as alturas estão em questão, é considerado retrógrado do primeiro: uma reavaliação, portanto, da técnica de "espelhamento" que já havia sido experimentado por Webern na Sinfonia Op. 21. O segundo movimento é, no entanto, a “interpretação livre” do anterior, uma vez que apresenta variações dentro da duplicação rígida das alturas através de uma rarefação progressiva do material sonoro. "Os dois movimentos, portanto, parecem complementares, onde o primeiro representa a continuidade e a adesão à estrutura rigorosa serial, embora pouco personalizado, o segundo dá uma maior

\footnotetext{
${ }^{6}$ Internationale Ferienkurse für Neue Musik

7 "The complex writing of the score, together with myriad of performing marks present on virtually every note, is evidence of the hard work and care that went into the writing of the Quartet, carried out in the conviction of having acquired a distinctive and personal language" (Russo, 2010, s/p).

8 "It has been an enormous job, much worse than writing the parts for an orchestral piece", and again "I believe that with this work I have truly found my path", and yet again "Everything that I have composed before is old 'kitsch'" (Maderna apud Russo, 2010, s/p).
} 
sensação de liberdade e um sentido 'lírico'” (Russo, 2010, p. VII)9 . Apesar de relativamente extensa a literatura crítica que lida com esta obra, o processo de composição que determina a estrutura ainda não parece ser claro. O Quarteto é um trabalho serialista, com base em 12 grupos de 12 notas. A identificação da série dentro da peça é, no entanto, apenas claramente possível no primeiro movimento, dado que, no segundo movimento, métodos de desgaste (erosion methods) são aplicados ao material, eliminando assim muitas alturas.

\section{O estudo experimental}

Foi feita uma comparação entre as análises macroformais do segundo movimento do Quarteto de Maderna, efetuadas por profissionais analistas italianos, e as percepções auditivas do movimento realizadas pelos dois grupos de sujeitos, músicos e não-músicos. A relação entre a memorização da macroforma e a percepção de tensão e relaxamento durante audição em tempo real foi também observada. As audições da obra foram realizadas na Itália (Bolonha, com estudantes universitários) e na Escócia (com estudantes da Universidade de Edimburgo).

\section{As "macroformas" dos analistas}

Várias tarefas foram dadas aos analistas profissionais para que eles pudessem realizar uma análise de partitura com base em critérios explícitos de percepção, tendo em conta a sua experiência pessoal de ouvir da peça. O analista deveria, no entanto, estar ciente das dificuldades de percepção e por problemas relacionados com a memória encontrados pelo ouvinte, por isso sua tarefa seria, precisamente, tornar esses problemas explícitos (ver também Cross, 1998). O analista deveria, portanto, considerar questões como: quais as dificuldades de percepção e

9 "The two movements thus appear complementary, where the first represents the continuity of and adherence to the rigorous serial structure, albeit somewhat personalized, while the second gives a greater sensation of freedom and a 'lyrical' sense" (Russo, 2010, p. VII). 
memorização pode o ouvinte encontrar na peça que eu estou analisando? Que estruturas podem ser claramente percebidas? Qual dessas estruturas pode ser memorizada?

As 4 macroformas foram obtidas por meio dos seguintes procedimentos:

$1^{\circ}$ ) Cada analista tinha autonomia com as seguintes etapas: a) ouvir a peça tantas vezes quanto ele necessitava; b) tentar e memorizar a macroforma, que é a obra inteira e, assim, as partes principais; c) analisar a macroforma memorizada com a ajuda da partitura, indicando na partitura os pontos de divisão das partes percebidas e memorizadas. 0 analista então precisava teorizar sobre o processo empreendido, apresentando algumas hipóteses sobre sua percepção própria, elaborando alguns critérios de análise da macroforma com base em sua experiência/escuta própria, esclarecendo estes critérios e descrevendo o método com o qual ele aplicou estes critérios para na análise da partitura. $2^{\circ}$ ) As análises foram coletadas, discutidas e verificadas por todos os analistas.

$\left.3^{\circ}\right)$ Apenas as análises que demostraram a coerência entre as hipóteses, critérios e métodos, foram aceitos para o experimento.

Cada analista estava livre para seguir suas próprias escolhas, usando as ferramentas teóricas e metodológicas que ele considerasse mais adequadas. Por esta razão, as macroformas obtidas foram vinculadas a diferentes teorias e abordagens, tais como a teoria Deliège de sugestão de abstração (cue abstraction), ou abordagem dinâmica de Imberty, ou tempo e critérios de segmentação em percepção.

Foi estabelecido que estas ferramentas deveriam servir para explicar cada experiência pessoal de audição, e que não deveriam ser utilizadas para expor teorias estáticas de composição por exemplo. O objetivo era não usar métodos teóricos de análise da macroforma, mas a hipótese de critérios de análise da macroforma tão próximo quanto possível das de um ouvinte. A diferença fundamental com relação à análise auditiva era de que os analistas podiam ouvir a peça muitas vezes. Desta forma, procurou-se obter macroformas de "especialistas" 
elaboradas por profissionais da análise musical com hipóteses analíticas provenientes da audição e não de teorias analíticas e estéticas.

As hipóteses de percepção, portanto, corroboraram com a experiência dos "puros" analistas. Este procedimento deu origem às seguintes macroformas, que foram selecionadas para serem comparadas com as macroformas memorizadas pelos ouvintes que participaram na fase experimental.

$\mathrm{Na}$ Macroforma 1, por exemplo, o analista que apresentou esta macroforma, hipotetizou que a sua percepção da macroforma, depois de ouvir a peça várias vezes, foi atribuída à percepção de "sinais" - como os definiu Deliège, que é "breve mas significativo e são estruturas significativas, que se destacam do plano de fundo sonoro" (Deliège 2007, p. 13) ${ }^{10}$ - e ao fenômeno dinâmico da percepção de tensão e relaxamento, como hipotetizado por Imberty (1981).

Em termos de análise estrutural, o autor desta macroforma indicou na partitura que a alternância da tensão/relaxamento foi devido principalmente a alternância de pistas "agressivas" (durações curtas, notas salientadas, tallone, tremolo, sforzzandi, pizzicattti, Detaché, textura densa, a intensidade de $m f$ para fff, sons de ruído, non vibrato), que prevalecem nas zonas de tensão, e pistas "suaves" (durações longas, Ponticello, col. legno, intensidade mp, ppppp, con sordina), predominantes nas zonas de relaxamento.

De acordo com este analista, esses traços recorrentes em determinadas seções e não em outras, também representam pistas que chamam a atenção do ouvinte através de sua repetição, permitindo que o ouvinte crie um mapa mental da obra, determinando, portanto, "imprinting", como definido por Deliège.

De acordo com este analista, a tensão/relaxamento também depende do comprimento das partes (isto é, quando as características extremas e leves seguem de uma a outra de perto, em um momento de resultados de tensão) e sobre as funções retóricas que algumas partes parecem sugerir (introdução, clímax, coda).

MACROFORMA 1 - Na Macroforma 1 a obra foi dividida em 7 partes: cada parte foi caracterizada por um certo grau de tensão (1, 2 ou 3), e um certo grau de relaxamento (1, 2 ou 3). Parte I, comp. 1-10 é caracterizado por um baixo

10 "Brief but meaningful and significant structures, which stand out from the sound background". 
nível de tensão (tensão 1), parte II, comp. 10-55, é caracterizada por um aumento da tensão (tensão 2), parte III, comp. 55-96, é caracterizada por relaxamento (relaxamento I); parte IV. comp. 97-117 é caracterizada pela média tensão (tension2); parte V, comp. 118-132, é caracterizada pela alta tensão (tensão 3); parte VI, comp. 132-173, é caracterizada por meio de relaxamento (relaxamento 2) e, finalmente, parte VII, comp. 174-199, é caracterizada pelo elevado relaxamento (relaxamento 3) (Ver Ex. 1).

MACROFORMA 2 - A macroforma 2 foi obtida a partir da macroforma 1. As duas primeiras partes (partes I e II) da macroforma 1 podem ser percebidas como uma peça única, em que a tensão aumenta (Tesão 1 -> Tensão 2), comp. 1 55 (tensão crescente), uma segunda parte do relaxamento, comp. 55-96 (relaxamento), correspondente a parte III da macroforma 1; parte III, comp. 97132, que agrupa partes IV e V da macroforma I, em que um aumento de tensão é percebida (parte superior da tensão); e, finalmente, parte IV, comp. 132-199 (relaxamento crescente), que agrupa as partes VI e VII da macroforma 1 (ver Ex. 1).

MACROFORMA 3 - Esta macroforma é baseada na hipótese de abstração de pistas de Deliège (2001a, 2001b) e divide a obra em 4 partes. Cada parte é caracterizada por um elemento muito perceptual: as partes I e III caracterizam-se por uma variabilidade interna; parte II é caracterizada pelo pulso regular desempenhado pelo violoncelo nos comp. 73-87; parte IV por sons suaves e possui função retórica como parte final. A variabilidade das partes I e III dependem da alternação de momentos "agressivos" (caracterizados por fortes, momentos "acentuados" e de longa duração), e "suaves" (caracterizados por momentos pedale, notas mais altas e suaves, fragmentos melódicos, sussurros). As quatro partes são organizadas em: parte I, comp. 1-72 (variabilidade); parte II, comp. 73-87 (tempo regular); parte III, comp. 88-131 (variabilidade); parte IV, comp. 132-199 (final "claro") (ver Ex. 1).

MACROFORMA 4 - Esta macroforma baseia-se em fortes segmentações, agrupamento de seções e repetição das seções. As seções são caracterizadas pela coerência interna. todas as partes da macroforma podem ser compostas de várias seções diferentes. Neste caso, a definição de macroforma é um pouco diferente do 
que aquela utilizada nas macroformas dos outros analistas. Cada parte é caracterizada pelo ritmo. A macroforma é constituída pela seguinte estrutura: parte I, comp. 1-62 (Allegro); parte II, comp. 63-96 (Adagio); parte III, comp. 97141 (Presto); parte IV, comp. 142-199 (II Adagio). A Tabela 1 mostra as 4 macroformas indicadas pelos analistas:

Tabela 1 - Macroformas dos analistas

\begin{tabular}{|c|c|c|c|c|}
\hline$M 1$ & $M 2$ & compassos & M3 & M4 \\
\hline $\begin{array}{l}\text { Parte I: tensão } \\
\text { baixa }\end{array}$ & \multirow{6}{*}{$\begin{array}{l}\text { Parte I: } \\
\text { Tensão crescente } \\
\text { (pistas de } \\
\text { sonoridade } \\
\text { "agressiva") }\end{array}$} & $1-10$ & \multirow{10}{*}{$\begin{array}{l}\text { Parte I variabilidade } \\
\text { interna (momentos de } \\
\text { sonoridade "agressiva") }\end{array}$} & \multirow[t]{8}{*}{ Parte I: Allegro } \\
\hline \multirow{5}{*}{$\begin{array}{l}\text { Parte II: tensão } \\
\text { média (pistas } \\
\text { de sonoridade } \\
\text { "agressiva") }\end{array}$} & & $11-20$ & & \\
\hline & & $21-30$ & & \\
\hline & & $31-40$ & & \\
\hline & & $41-50$ & & \\
\hline & & $51-55$ & & \\
\hline \multirow{8}{*}{$\begin{array}{l}\text { Parte III: baixo } \\
\text { relaxamento } \\
\text { (pistas "sua- } \\
\text { ves") }\end{array}$} & \multirow{8}{*}{$\begin{array}{l}\text { Parte II: } \\
\text { relaxamento } \\
\text { (pistas “suaves") }\end{array}$} & $56-60$ & & \\
\hline & & $61-62$ & & \\
\hline & & $63-70$ & & \multirow[t]{6}{*}{ Parte II: Adagio } \\
\hline & & $71-72$ & & \\
\hline & & $73-80$ & \multirow[t]{2}{*}{ Parte II: pulso } & \\
\hline & & $81-87$ & & \\
\hline & & $88-90$ & \multirow{8}{*}{$\begin{array}{l}\text { Parte III: variabilidade } \\
\text { interna (momentos de } \\
\text { sonoridade "agressiva") }\end{array}$} & \\
\hline & & $91-96$ & & \\
\hline \multirow{3}{*}{$\begin{array}{l}\text { Parte IV: } \\
\text { média } \\
\text { tensão (pistas } \\
\text { de sonoridade } \\
\text { "agressiva") }\end{array}$} & \multirow{6}{*}{$\begin{array}{l}\text { Parte III. Máxima } \\
\text { tensão (pistas de } \\
\text { “sonoridade } \\
\text { agressiva”) }\end{array}$} & $97-100$ & & \multirow[t]{8}{*}{ Parte III: Presto } \\
\hline & & $101-110$ & & \\
\hline & & $111-117$ & & \\
\hline \multirow{3}{*}{$\begin{array}{l}\text { Parte V: tensão } \\
\text { máxima (pistas } \\
\text { de sonoridade } \\
\text { "agressiva") }\end{array}$} & & $118-120$ & & \\
\hline & & $121-130$ & & \\
\hline & & $131-132$ & & \\
\hline \multirow{6}{*}{$\begin{array}{l}\text { Parte VI: } \\
\text { médio relaxa- } \\
\text { mento (pistas } \\
\text { "suaves”) }\end{array}$} & \multirow{9}{*}{$\begin{array}{l}\text { Parte IV: aumento } \\
\text { de relaxamento } \\
\text { (pistas "suaves") }\end{array}$} & $133-140$ & \multirow{9}{*}{$\begin{array}{l}\text { Parte IV: Final } \\
\text { (momento "suave") }\end{array}$} & \\
\hline & & 141 & & \\
\hline & & $142-150$ & & \multirow{7}{*}{ Parte IV: Adagio II } \\
\hline & & $151-160$ & & \\
\hline & & $161-170$ & & \\
\hline & & $171-173$ & & \\
\hline \multirow{3}{*}{$\begin{array}{l}\text { Parte VII: } \\
\text { relaxamento } \\
\text { máximo } \\
\text { (pistas } \\
\text { "suaves") }\end{array}$} & & $174-180$ & & \\
\hline & & $181-190$ & & \\
\hline & & 191-199 & & \\
\hline
\end{tabular}




\section{Testes dos ouvintes}

Participantes. Dois grupos de indivíduos tomaram parte no experimento: 25 nãomúsicos e 32 músicos. O experimento foi realizado tanto em Bolonha (10 nãomúsicos, 12 músicos) como em Edimburgo (15 não-músicos, 20 músicos).

Material para audição: O "Quartetto per archi in due tempi", $2^{\circ}$ movimento, de Bruno Maderna (1955); duração 65'7"; executado pelo Ex Novo Ensemble (CD STR Stradivarius 33330).

Equipamento: foi utilizado um programa de computador especial chamado EPM (Experiências sobre a percepção da música), criado na Universidade de Pádua. Este programa permite que cada sujeito ao ouvir a peça através de fones de ouvido possa indicar o ponto de divisão percebida enquanto escuta em tempo real elementos de tensão/relaxamento, deslocando o mouse para cima (tensão) e para baixo (relaxamento).

Procedimentos. Para cada indivíduo foi alocado um computador e dado um questionário, que, para além das respostas escritas, continha as tarefas a serem realizadas utilizando o programa de EPM. O questionário também continha detalhes de como usar o programa. O experimento foi realizado em uma sala com vários computadores. Os sujeitos foram divididos em várias sessões de grupos mistos (músicos e não-músicos) de 5, 10, 15 pessoas. Um operador lia as instruções gerais para a utilização do questionário. Se necessário, dois assistentes estavam disponíveis para resolver problemas individuais. Cada experimento durou cerca de 60 minutos, envolvendo várias etapas e tarefas.

Depois de uma escuta inicial - a fim de familiarizar os participantes com a peça e com o programa de computador (tarefa 1) - os sujeitos tiveram que ouvir a peça duas vezes e identificar suas seções principais (tarefa 2). Eles foram orientados pela seguinte ordem: “Ouça a peça mais uma vez: Na sua opinião, em quantas partes principais a obra pode ser dividida? Por favor, indique o comprimento de cada parte ao longo desta linha horizontal que representa a totalidade da duração da peça. Certifique-se se o local indicado é proporcional ao comprimento de cada parte. Só marcar o princípio, as seções mais substanciais e não os trechos mais curtos. O número total de partes não deve ser inferior a dois e 
não pode ser superior a 8". No decorrer das duas audições seguintes, eles tinham de indicar os pontos de divisão das seções no computador usando o programa de EPM e após a segunda audição, eles deveriam salvar os resultados (tarefa 3). Nesta etapa a ordem era: "Ouça a peça novamente e tente marcar no computador as partes que você indicou, marcando os pontos de divisão com o mouse".

Nas próximas tarefas 4 e 5 os indivíduos tinham de indicar os adjetivos e tipo de sons que eles acreditavam que eram característicos de cada seção. Para a tarefa 4 a orientação foi a seguinte: "Por favor, assinale os adjetivos que você acha que caracteriza cada seção. Você pode indicar qualquer número de adjetivos para cada seção, e você também pode adicionar um novo adjetivo". A lista de adjetivos foi a seguinte: suave, alegre, violento, tenso, lento, relaxante, variável.

Para a tarefa 5 a ordem dada foi a seguinte: "Por favor, marque os tipos de sons que caracterizam cada seção. Você pode indicar quantos você quiser, e você também pode adicionar um novo tipo de som". A lista de sons foi a seguinte: poucos sons, sons repetidos regularmente perto uns dos outros, sons longos, sons fracos, sons suaves, sons fortes, sons acentuados, silêncios, muitos sons.

Nas tarefas 6 e 7 os participantes tiveram que indicar as maiores zonas de tensão e relaxamento por meio do deslocando do mouse. Para a tarefa 6, eles foram convidados a realizar a seguinte função: "Ouça a peça novamente, tentando determinar as maiores áreas de tensão e relaxamento. As áreas de tensão serão caracterizadas por instabilidade, como a ação de inspirar, as áreas de relaxamento serão caracterizadas pela tendência para uma situação estável, como expirar". Para a tarefa 7, eles foram convidados a fazer o seguinte: “Depois de ter determinado estes momentos, marque-os no computador, movendo o mouse: quando você perceber maiores áreas de tensão, caracterizado pela instabilidade, como inspirar, mova a bolinha para cima usando o mouse; quando você perceber maiores áreas de relaxamento, que tendem para uma situação estável, como expirar, mova para baixo a bolinha. Lembre-se que the é apresentada uma escala de 1 a 100 , visualizada na tela. Você pode ouvir duas vezes, a segunda vez que você deve salvar os resultados". Os resultados foram registrados movendo fisicamente o mouse para cima ou para baixo, correspondendo à tensão ou relaxamento 
percebido no momento de audição. Esta foi automaticamente registrada em uma escala 1-100 visível na tela. Após a escuta, eles salvaram os resultados (tarefa 5).

Hipóteses experimentais: 1. As macroformas percebidas pelos sujeitos estão correlacionadas às macroformas indicadas pelos analistas; 2. existe uma correlação entre macroformas e tensão/relaxamento percebidos pelos sujeitos; 3. finalmente, a diferença entre os músicos e não músicos não é significativa.

\section{Resultados}

Este artigo mostra os resultados de tarefas 3 e 7. De um modo geral, os resultados mostram que as percepções de macroformas dos sujeitos eram extremamente heterogêneas. A maioria dos sujeitos, sejam músicos ou não músicos, indicaram mais divisões do que aqueles usadas como hipóteses pelos analistas. Primeiro foram consideradas como similar ou não as macroformas propostas pelos ouvintes (tarefa 3) com aquelas sugeridas pelos analistas musicais. As respostas dadas na tarefa 3 foram agrupadas em vários intervalos (ver Figura 1) correspondentes às áreas onde os temas foram identificados como pontos de divisão da obra. A Figura 1 não mostra pontos precisos, mas sim a variedade de posições no gráfico. Os intervalos mostram o espaço de tempo em que um conjunto de respostas é agrupado. Um índice empírico foi desenvolvido para verificar quão perto ou longe, as macroformas percebidas pelos participantes foram com aquelas propostas pelos analistas. Foram considerados todos os pontos de divisão dados para cada macroforma percebida e foi feita a comparação com as macroformas dos analistas.

A seguinte fórmula foi aplicada: $I=\left(c^{*} 100\right) / A$, na qual "c" é o número de pontos que coincidem com os pontos nas macroformas dos analistas e A é o número de todos os pontos de divisão indicados pelos sujeitos. Dado que em M1 há mais pontos de divisão do que em M2, M3 e M4, para o cálculo de I, para cada macroforma, foi assumido que $\mathrm{A}$ não poderia ser menor do que o número dos pontos de propostas nas macroformas dos analistas. Este ajuste também evitou a divisão por erro zero. Os dados indicaram que o M2 foi o mais 
semelhante e M3 o mais divergente em relação às macroformas percebidas pelos ouvintes. A diferença entre os meios de correspondência relativa $M 1$ e $M 3$ são estatisticamente significativas de acordo com o teste de Wilcoxon ( $p<0,001)$; diferença entre as médias das macroformas 1 e 4, 2 e 3, 2 e 4, 3 e 4 são também estatisticamente significativas (respectivamente $p<0,01, p<0,001$, $p<0,01$ e $p$ $<0,05$ de acordo com a teste Wicoxon). Não foram encontradas diferenças significativas entre as médias de correspondência relativa $M 1$ e $M 2$. Não houve diferenças significativas no meio de correspondência sobre as variáveis independentes - formação musical e nacionalidade - de acordo com o teste de Mann-Whitney. Todos estes resultados estão resumidos na Tabela 2.

Tabela 2- Índice de Correspondência ${ }^{11}$ das macroformas propostos pelos analistas musicais e macroformas percebidas pelos outros participantes de acordo com a sua nacionalidade e formação musical

\begin{tabular}{|l|l|l|l|l|l|l|}
\hline \multicolumn{1}{|c|}{ Macroformas } & \multicolumn{2}{c|}{ Escoceses } & \multicolumn{2}{c|}{ Italianos } & \multicolumn{2}{c|}{ Todos os sujeitos } \\
\hline $\begin{array}{l}\text { Meios de correspon- } \\
\text { dência }\end{array}$ & Músicos & Não Músicos & Músicos & Não Músicos & $\begin{array}{l}\text { Músicos e } \\
\text { não Músicos }\end{array}$ & $\begin{array}{l}\text { Desvio } \\
\text { padrão }\end{array}$ \\
\hline Macroforma 1 & 37.4 & 35.9 & 33.9 & 33.1 & 35.6 & 18.8 \\
\hline Macroforma 2 & 30.7 & 39.1 & 40.1 & 51.7 & 37.8 & 23.4 \\
\hline Macroforma 3 & 16.3 & 7.2 & 14.6 & 9.3 & 13.0 & 18.3 \\
\hline Macroforma 4 & 22.1 & 21.7 & 18.1 & 13.6 & 19.8 & 14.9 \\
\hline
\end{tabular}

Não foi encontrada uma correspondência elevada entre as macroformas percebidas e macroformas analisadas. No entanto, é possível observar uma tendência geral que confirma os pontos de divisão indicados pelos analistas em $M 1$ e $M 2$. Pode ser visto a partir da Tabela 2 que $M 1(I=35,6)$ e o derivado $M 2$ $(I=37,8)$ são os mais próximos às macroformas percebidas por ambos os músicos (M1: I = 33,9 italiana e I = 37,4 escocês; $M 2$ : I = 40,1 italiano e I = 30,7 escocês) e não-músicos ( $M 1: I=33,1$ italiano e $I=35,9$ escocês; $M 2: I=$ 51,7 italiano e I = 39,1 escocês). Os resultados para M4 são decididamente inferiores $(I=19,8)$, enquanto $M 3$ é a mais distante das macroformas percebidas

\footnotetext{
11 Index of Correspondence is an empirical measure that we have adopted given by the number of the points coincident with macroforms $(1,2,3$ and 4) multiplied by 100 and divided by the number of all the points given by the subjects to define their macroforms.
} 
$(I=13,0)$, tanto para os músicos ( $I=14,6$ italiano e $I=16,3$ escocês) quanto para não-músicos ( $(=$ 9,3 Italiano e $I=7,2$ escocês).

Estes resultados parecem indicar que os critérios utilizados em análises de M1 e M2, isto é, o processo de sinalização de abstração e o processo dinâmico associado à percepção de tensão e relaxamento, pode ser preditivo da análise auditiva realizada em tempo real durante a audição e poderia orientar o analista durante a análise da partitura.

O resultado permite confirmar os resultados obtidos por Deliège em seus estudos experimentais. Mas os resultados de M3 e M4 são ainda mais interessantes. Com efeito, dado que a M3, a mais distante macroforma percebida pelos ouvintes, também foi baseada na teoria de Deliège (cue theory), pode-se considerar hipoteticamente que pode existir uma contradição entre os resultados obtidos. Na realidade, a explicação pode ser a seguinte: em M3 foram determinadas partes I e III com base na sua variabilidade interna que foi dada pela simultaneidade de elementos "agressivos" e elementos "suaves".

Os sujeitos, por outro lado, parecem ter preferido agrupar de acordo com a percepção de "similaridade", em vez da variabilidade interna de cada uma das partes, separando assim os momentos caracterizados pela repetição de elementos "agressivos" para momentos caracterizados pela repetição de elementos suaves. $M 3$, portanto, não poderia ser previsível na análise auditiva, porque embora referindo-se ao modelo Deliège, não foi respeitado o princípio da semelhança. Esta conclusão é particularmente importante porque destaca a importância da percepção de semelhança nos processos cognitivos de memorização e processamento musical, como confirmado por diferentes perspectivas em diversos estudos publicados recentemente (Toiviainen 2007).

Além disso, a partir da Figura 1, é claro que a divisão posicionada no compasso 73, que indica o início da segunda parte, baseada no pulso rítmico, era completamente irrelevante a partir de um ponto de vista perceptual. No que diz respeito ao $M 4$, pode-se observar que os dois critérios de análise utilizados para esta macroforma, isto é, fortes segmentações destinadas a servir de descontinuidade local, e a indicação de tempo (Allegro, Adagio, etc), mostram uma utilização direta de parâmetros teóricos em vez de parâmetros do tipo 
perceptivo. Este aspecto poderia justificar a baixa correlação com as macroformas percebidas como M4. Além disso, este resultado poderia confirmar a distinção teórica inicial, segundo a qual as descontinuidades locais não são necessariamente percebidas como divisões de peças e não são, por conseguinte, determinantes para a memorização da macroforma.

Este resultado foi sustentado pelos seguintes dados analíticos que mostraram uma correlação entre as tensões percebidas e os pontos de divisão indicados por parte dos ouvintes (tal como explicado no parágrafo seguinte).

Gráfico 1- A frequência dos pontos de divisão indicado pelos ouvintes (dois pontos negros) em comparação com os pontos de divisão indicados pelos analistas (M1, M2, M3, M4).

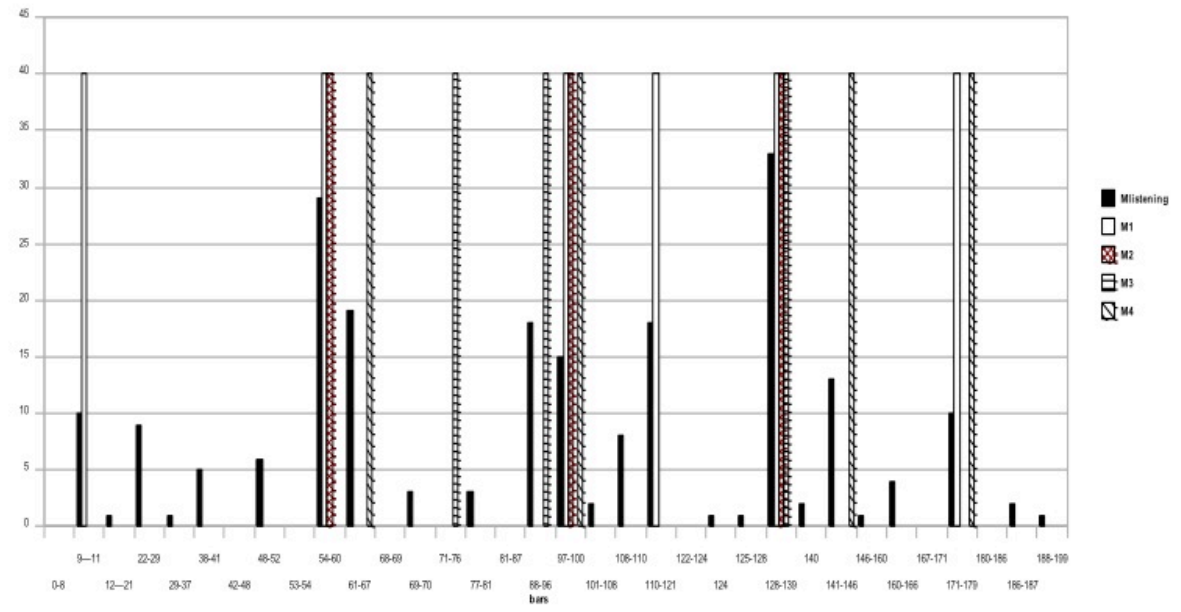

De modo a obter o ponto de divisões indicadas pelos ouvintes, as respostas dadas na tarefa 3 foram agrupados em diversas escalas correspondentes para a zona onde os indivíduos identificaram os pontos de divisão da peça. A Figura 1 não mostra pontos precisos, mas sim a variedade de posições na obra. Os intervalos, portanto, mostram o espaço de tempo em que um conjunto de respostas é agrupado. 


\section{Tensão e relaxamento}

Foram examinadas as relações entre tensão/relaxamento e as macroformas. Considerando todos os pontos de divisão indicados pelos sujeitos (ver Figura 1), os pontos em que as tensões foram indicadas e os pontos quando os relaxamentos foram indicados, foi possível verificar que há uma correlação positiva e altamente significativa entre os pontos de divisão e tensão. Estes resultados encontram-se resumidos na Tabela 3.

Tabela 3- Coeficiente de correlação entre os Pontos de divisão e Análise de tensão/Relaxamento

\begin{tabular}{|l|c|c|c|}
\hline & Pontos de divisão & Tensão & Relaxamento \\
\hline Pontos de divisão & 1.00 & $.7639^{* *}$ & .0562 \\
\hline Tensão & $.7639^{* *}$ & 1.00 & -.1483 \\
\hline Relaxamento & .0562 & -.1483 & 1.00 \\
\hline
\end{tabular}

$* \mathrm{p}<.05 * * \mathrm{p}<.01 \quad$ (two tailed)

Finalmente, foram verificadas as correlações entre os pontos de tensão, divisão e relaxamento (3 variáveis, 2 d.f.). Os resultados da regressão linear múltipla indicaram que tanto a tensão e relaxamento são preditivos de pontos de divisão, assim como os valores de beta muito mais elevados, quando a tensão é considerada a variável independente, como se pode ver na Tabela 4.

Não foram observadas diferenças significativas entre os diferentes grupos de indivíduos (italianos e escoceses, músicos e não-músicos): este fenômeno, portanto, não depende de treinamento musical. Pode-se argumentar que uma alteração da ordem das tarefas poderia ter influenciado estes resultados. Parte-se do pressuposto de que as tarefas 4 e 5 podem ter gerado alguma distração em relação às tarefas 3 e 7 . De qualquer modo, o resultado obtido precisaria ser confrontado com os resultados de um protocolo experimental em que a ordem das tarefas é modificada (através da realização de teste de tensão/relaxamento antecipados, por exemplo). 
Tabela 4- A regressão linear múltipla

\begin{tabular}{|l|l|l|l|}
\hline Variável dependente & Variável independente & Beta & $\mathrm{P}$ \\
\hline Pontos de divisão & Tensão & .783102 & .0000 \\
\hline Tensão & Relaxamento & .170434 & .0156 \\
\hline
\end{tabular}

Nas Macroformas 1 e 2 são propostas algumas características estruturais para explicar a alternância de tensão e relaxamento: pistas "agressivas" (como curtas, notas salientadas, tallone, tremolo, sforzzandi, pizzicattti., Detaché, textura densa, a intensidade de $m f$ para $f f f$, sons de ruído, non vibrato, que caracterizam o momento de tensão) contra as pistas "ligth" (durações longas, Ponticello, col. legno, intensidade mp, ppppp, con sordina) que caracterizam o momento de relaxamento. Pode-se observar que a organização sonora de timbre e densidade do som é importante, assim como é a organização da duração.

\section{Conclusão}

O principal objetivo deste estudo foi investigar as relações entre as macroformas percebidas pelos indivíduos enquanto escuta de uma obra pós-tonal e as macroformas da mesma peça indicadas por analistas profissionais com base na escuta e não em teorias analíticas. Foi observado que as macroformas dos analistas, com base no modelo de pistas de abstração, sobre a experiência dinâmica de tensão e relaxamento, são as mais semelhantes às macroformas percebidas pelos ouvintes experimentais.

Além disso, foi verificada uma correlação significativa entre as macroformas auditivas e a experiência de tensão/relaxamento percebidas durante a escuta em tempo real. Nota-se também que, enquanto grupos de diferentes níveis hierárquicos estão presentes no estudo de Deliège, neste estudo foi limitado - dentro do âmbito da investigação - para a percepção de partes mais extensas (maiores). Mesmo se for isolado esse nível, as alternância das pistas "agressivas" e "suaves" parecem funcionar como invariantes "implícitas" que se repetem, alternativamente, e isto permitiu a percepção de agrupamentos grandes e, 
portanto, a formação de uma impressão na memória do ouvinte. No caso do quarteto de Maderna, tais semelhanças parecem ser determinadas por timbres particulares utilizados pelas cordas, que são elementos que têm um tom prevalentemente rico, conforme indicado pelos analistas tanto em $M 1$ quanto em M2, este aspecto parece estar de acordo com Lerdahl (1989).

O processo de escuta e da memória da forma musical parece estar diretamente ligado à dimensão dinâmica através da qual a obra é experimentada pela pessoa em processo de escuta em tempo real, e isso contribui de forma significativa para a experiência da forma, mesmo quando as referências tonais estão ausentes. Este resultado está de acordo com Imberty (2005) e Lalitte et al. (2009) e com a experiência anterior da autora (Addessi e Caterina, 2000). Finalmente, não foi observada diferença significativa entre os músicos e não músicos. Este resultado confirma os estudos experimentais realizados por Deliège, Imberty, Bigand e estudos anteriores realizados pela autora e colaboradores. Estes critérios podem ser utilizados de forma explícita pelos analistas para a análise da partitura (Cruz, 1998). Os objetivos e os meios empregados por alguém que estuda análise da partitura (musicólogos, compositores, analistas) são diferentes dos utilizados no estudo da análise auditiva (processo psicológico na música) (ver Clarke 1989, Sloboda 2005). No entanto, pode-se pensar que alguns procedimentos analíticos são comuns em ambas as abordagens, tais como as experimentadas no estudo apresentado neste artigo - a divisão em partes que foi definida como macroforma. A distinção entre a competência "implícita" do ouvinte e da conceituação explícita do analista ainda é válida, mesmo sob essas condições. O analista tende a pensar em categorias conceituais, transformando a percepção categórica ou simplesmente uma percepção que obedece a leis gerais cognitivas, em conceitos definidos, até mesmo a ponto de colocá-los em áreas onde tais conceitos ainda não foram definitivamente codificados por meio da teoria musical.

Outra diferença essencial entre o ouvinte e o analista verificada neste estudo foi a de que o tempo, para este último, sempre foi livremente reversível. Em uma partitura musical, o tempo é transformado em um espaço onde é possível 
parar em qualquer ponto. Além disso, como apontou Cook (1990), a percepção visual da partitura também pode afetar a análise do analista e sua escuta.

Uma consideração final pode ser feita ligando os resultados experimentais obtidos com uma visão estética do quarteto de Maderna. Esta obra é realmente especial, mas também típica do estilo pós-tonal. Parece não ter uma macroforma pois as pistas perceptivas dividem a peça em várias seções muito pequenas: às vezes estas seções duram apenas alguns segundos e o resultado perceptivo não é uma macroforma, mas uma sensação de fragmentação. As descontinuidades locais parecem ser dominantes. Por outro lado, os resultados relativos à articulação dinâmica de tensão e de relaxamento e a teoria do “imprinting" permitiram explicar porque é possível perceber grandes e coerentes seções, apesar das fortes descontinuidades locais ${ }^{12}$. O resultado é um quarteto o qual que "a recusa de qualquer herança morfológica e graça formal sutil, existem lado a lado, junto com a crença em uma ordem pré-estabelecida de série e da necessidade de improvisação" (Berio 1957, s/p) ${ }^{13}$.

Agradecimentos $^{14}$

\footnotetext{
${ }^{12}$ Between these two levels, one a macroformal level made up of large sections and the other a fragmentary micro-structural level, a middle level also appears, characterised by the presence of "stylistic figures" such as melodic figures, "echo", etc. This intermediate level, which we can define as intertextual, creates a series of references which are external to the work (Deliège would define them as external similarities; "extraopus topics" according to Agawu 1991). The three levels seem to dialogue with each other in the Maderna quartet. A new experimental study could show how the listener could experience an interweaving of these three levels. Even if the theoretical references are different, the experimental studies carried out by Krumhansl $(1996,2009)$ on how the perception of musical ideas can guide the mental representation of a piece during real time listening, suggest a possible path that could be taken in this direction.

13 "refusal of any morphological legacy and subtle formal grace exist side by side, together with belief in a pre-established serial order and the need for improvisation." (Berio 1957).

${ }^{14}$ AGRADECIMENTOS: Obrigado a Christian Temporali do programa EPM; Prof. Roberto Caterina da Universidade de Bolonha, pelas análises estatísticas; Christina Anagnostopoulou e Miguel Ferrand pela colaboração durante a administração do teste para os estudantes de Edimburgo; Arquivo Maderna da Universidade de Bolonha, por fazer a gravação de áudio e disponibilizar a partitura do Quartetto de Maderna; Marco Russo por gentilmente me ter permitido o acesso à pré-impressão da nova edição crítica do Quartetto de Maderna.
} 


\section{Referências}

ADDESSI, A. R.; CATERINA, R. Perceptual musical analysis: Segmentation and perception of tension. Musicæ Scientiæ, 4/1, 2000, p. 31-54.

Perception of the macroform in the Quartetto per Archi in due tempi (1955) by Bruno Maderna. In Second International Conference: Understanding and Creating Music. Caserta, Seconda Università degli Studi di Napoli , 2002, cd-rom.

Analysis and perception in post-tonal music: an example from Kurtág's String Quartet Op. 1., Psychology of Music, 33/1, 2005, p. 94-116.

AGAWU, K. Playing with signs. A semiotic interpretation of classic music. Princeton: Princeton University Press, 1991.

BAMBERGER, J. Coming to hear in a new way. In R. Aiello, J.A. Sloboda (Eds.), Musical Perception (p. 132-151). New York: Oxford University Press, 1994.

BARONI, M. The macroform in post-tonal music. Listening and analysis. Musicae Scientiae, 7/2, 2003, p. 219-240.

BARONI, M.; DALMONTE, R. (Eds) Bruno Maderna, documenti, Milano: Suvini Zerboni, 1995.

BERIO, L. Bruno Maderna: Quartetto in due tempi, in Concerti degli incontri musicali, quarto concerto, Notes for the concert, Milano, Sala Piccola del Conservatorio, 2 marzo 1957.

BIGAND, E. L'organisation perceptive d'oeuvres musicales tonales, Paris: Maison des Sciences de l'Homme, 1993.

Perceiving musical stability: the effect of tonal structure, rhythm, and musical expertise. Journal of Experimental Psychology: Human Perception and Performance, 23/3, 1997, p. 808-822.

More about the musical expertise of musically untrained listeners. Annals of the New York Academy of Sciences, 999, 2003, p. 1-10.

BIGAND, E.; PARNCUTT, R.; LERDHAL, F. Perception of musical tension in short chord sequences: The influence of harmonic function, sensory dissonance, horizontal motion, and musical training. Perception \& Psychophysics, 58/1, 1996 , p. $125-141$.

BIGAND, E.; POULIN-CHARONNAT, B. Are we "experienced listeners"? A review of the musical capacities that do not depend on formal musical training. Cognition, 100, 2006, p.100-130.

CROSS, I. Music analysis and music perception. Music Analysis, 17/1, 1998, p. 0320. 
DELIÈGE, I. A perceptual approach to contemporary musical forms. In S. McAdams and I. Deliège (Eds.), Music and Cognitive Sciences. Contemporary Music Review, 4, 1989, p. 213-230.

Wagner "Alte Weise": Une approche perceptive. Musicae Scientiae, Special Issue, 1998, p. 63-90.

Introduction: Similarity Perception $<->$ Categorization $<->$ Cue Abstraction. Music Perception, 18/3, 2001a, p. 233-243.

Prototype effects in music listening: An empirical approach to the notion of imprint. Music Perception, 18/3, 2001b, p. 371-403.

Similarity relation in listening to music: How do they come into play?. Musicae Scientiae, Discussion Forum 4A, 2007, p. 9-37.

DELIĖGE, I. AND EL AHMADI, A. Mechanisms of cue extraction in musical groupings: A study of perception on Sequenza VI for viola solo by L. Berio. Psychology of Music, 18/1, 1990, p. 18-44.

DIBBEN, N. The perception of structural stability in atonal music: The influence of salience, stability, horizontal motion, pitch commonality, and dissonance. Music Perception, 16/3, 1999, p. 265-294.

HABIB, M., BESSON, M. What do music training and musical experience teach us about brain plasticity?. Music Perception, 26/3, 2009, p. 279-285.

HASTY, Ch. Segmentation and process in post-tonal music. Music Theory Spectrum, 3, 1981, p. 54-73.

IMBERTY, M. L'occhio e I'orecchio. Sequenza III di Berio. In L. Marconi and G. Stefani (Eds.), I/ senso in musica, p. 163-186. Bologna: Clueb, 1987.

How do we perceive atonal music? Suggestions for a theoretical approach. Contemporary Music Review, 9, 1993, p. 325-338.

La Musique creuse le temps. Paris: L'Harmattan, 2005.

KRUMHANSL C. A perceptual analysis of Mozart's Piano Sonata K 282: Segmentation, tension, and musical ideas, Music Perception, n.13, 1996.

Musical tension: Cognitive, motional and emotional aspects. In:

Third Triennal ESCOM Conference. Proceedings, Uppsala: Uppsala University, 1997, cd-rom.

Plink: Thin Slices in Music. Oral presentation at the $7^{\text {th }}$ Conference of ESCOM, University of Jyväskylä, Finland, August 12-16, 2009.

LALITTE, P., BIGAND, E., MARTYNUSKA, K., DELBÉ, C. On listening to atonal variants of two piano sonatas by Beethoven. Music Perception, 26/3, 2009, p. 223-234.

LA RUE, J. Guidelines for style analysis. New York: Norton and Company, 1970.

LERDAHL, F. Atonal prolongation structure. In S. McAdams and I. Deliège (Eds.), Music and Cognitive Sciences. Contemporary Music Review, 4, 1989, p. 65-88. 
63.

Calculating tonal tension. Music Perception, 13/3, 1996, p. 319-

Spatial and psychoacoustic factors in atonal prolongation. Current Musicology, 63, 1999.

LERDAHL, F. AND JACKENDOFF, J. A generative theory of tonal music. Cambridge, Mass: MIT Press, 1983.

MEYER, L. B. Emotion and Meaning in Music, Chicago: University of Chicago Press, 1956.

MURAIL, T. "Esplorando il suono". Interview by A. R. Addessi, // Giornale della Musica, n. 185, vol. 2, 2002.

NIELSEN, F. Musical 'tension' and related concepts. In Th. Sebeok, J. UmijerSebeok (Eds.), Basic Concepts of Musical Signification. Special Issue of THe Semiotic Web, Berlin: Mouton, 1989.

PANTEV, C., ENGELIEN, A., CANDIA, V., ELBERT, T. Representational cortex in musicians: Plastic alterations in response to musical practice. In R.J. Zatorre, I. Peretz (Eds.), The biological foundations of music (p. 300-314). New York Accademy of Sciences, New York, 2001.

PASCUAL-LEONE, A. The brain that plays music and is changed by it. In R.J. Zatorre, I. Peretz (Eds.), The biological foundations of music (p. 315-329). New York: Accademy of Sciences, 2001.

RUSSO, M. Introduction. In B. Maderna, Quartetto per Archi in due tempi. Milano: Suvini Zerboni, 2010.

RUWET N. Méthodes d'analyse en musicologie. Revue belge de Musicologie, n.20, 1966, p. 65-90.

SCHLAUG, G. \& CHEN, C. The brain of musicians: A model for funziona and structural adaptation. In R.J. Zatorre, I. Peretz (Eds.), The biological foundations of music (p. 281-299). New York: Accademy of Sciences, 2001.

SLOBODA, J.A. Exploring the musical mind: Cognition, emotion, ability, function. New York: Oxford University Press, 2005.

TOIVIAINEN, P. (Ed.) Similarity perception in listening to music. Musicae Scientiae, Discussion Forum A/B, 2007. 